\title{
Bone Segmentation and 3D Visualization of CT Images for Traumatic Pelvic Injuries
}

\author{
Jie Wu, ${ }^{1}$ Ashwin Belle, ${ }^{4,7}$ Rosalyn H Hargraves, ${ }^{2}$ Charles Cockrell, ${ }^{3,4}$ Yang Tang, ${ }^{3}$ \\ Kayvan Najarian ${ }^{5,7}$ \\ ${ }^{1}$ Department of Computer Science, Virginia Commonwealth University, VA \\ ${ }^{2}$ Department of Electrical and Computer Engineering, Virginia Commonwealth University, VA \\ ${ }^{3}$ Department of Radiology, Virginia Commonwealth University, VA \\ ${ }^{4}$ Virginia Commonwealth University Reanimation Engineering Science Center, Virginia \\ Commonwealth University, VA \\ ${ }^{5}$ Department of Emergency Medicine, University of Michigan, MI \\ ${ }^{6}$ Department of Computational Medicine and Bioinformatics, University of Michigan, MI \\ ${ }^{7}$ Michigan Center for Integrative Research in Critical Care (M-CIRCC), University of Michigan, MI
}

Received 15 October 2012; accepted 17 October 2013

\begin{abstract}
Pelvic bone segmentation is a vital step in analyzing pelvic CT images, which assists physicians with diagnostic decision making in cases of traumatic pelvic injuries. Due to the limited resolution of the original CT images and the complexity of pelvic structures and their possible fractures, automatic pelvic bone segmentation in multiple CT slices is very difficult. In this study, an automatic pelvic bone segmentation approach is proposed using the combination of anatomical knowledge and computational techniques. It is developed for solving the problem of accurate and efficient bone segmentation using multiple consecutive pelvic CT slices obtained from each patient. Our proposed segmentation method is able to handle variation of bone shapes between slices there by making it less susceptible to inter-personal variability between different patients' data. Moreover, the designed training models are validated using a crossvalidation process to demonstrate the effectiveness. The algorithm's capability is tested on a set of 20 CT data sets. Successful segmentation results and quantitative evaluations are present to demonstrate the effectiveness and robustness of proposed algorithm, well suited for pelvic bone segmentation purposes. (c) 2014 Wiley Periodicals, Inc. Int J Imaging Syst Technol, 24, 29-38, 2014; Published online in Wiley Online Library (wileyonlinelibrary.com). DOI: 10.1002/ima.22076
\end{abstract}

Key words: traumatic pelvic injury; bone segmentation; 3D visualization; registered active shape model

Correspondence to: Ashwin Belle, e-mail: bellea@umich.edu

Grant sponsor: National Science Foundation, Grant number: IIS0758410

\section{INTRODUCTION}

Traumatic pelvic injuries are often high energy injuries that constitute a major cause of death in trauma patients. Every year, traumatic pelvic injuries contribute to cases of death and permanent disability. According to the centers for disease control and prevention (CDC), trauma injury kills more people between the ages of 1 and 44 than any other disease or illness (CDC Report, 2009). On average, 15 U.S. workers die each day from traumatic injuries; simultaneously, thousands of U.S. workers visit emergency rooms for treatment of work-related traumatic injuries every day (Bureau of Labor Statistics, 2012). More than 300 patients with pelvic fractures were seen in less than 3 years at the University of Maryland Shock Trauma Center (Young et al., 1986), and patients with pelvic bone fractures who are present in shock have a mortality of 30-50\% (http://www.trauma.org/index.php/main/article/668/). When combined with injuries in other body regions, for example, the abdomen, the chance of mortality rises even higher, approaching $100 \%$ in some cases (http:// www.trauma.org/index.php/main/article/668/). Among different types of trauma with a high impact on the lives of Americans, traumatic pelvic injuries, caused mainly by sports, falls and motor vehicle accidents contribute to a large number of mortalities every year (Ali et al., 2008; Schiff et al., 2008). Traumatic pelvic injuries and associated complications, such as severe hemorrhage, multiple organ dysfunction syndrome (MODS), and blood clots traveling to the brain or lungs, result in the mortality rate from 8.6 to 50\% (UMD, 2005). 
Physicians treating traumatic pelvic injuries, especially pelvic bone fractures, utilize pelvic computed tomography (CT) images as an important resource for assessing the severity and developing prognosis of such injuries (Ji et al., 2009). For analysis the of pelvic CT images of injured patients, segmentation of the pelvic bone from the CT image is a vital step. It can be used to assess and evaluate ailments, measure how severe the injury is, provide quantitative measurement of bone fracture and detect the hemorrhage position around the fractured bones.

Image segmentation is an active research topic in the field of medical image processing. Various approaches such as thresholding, region growing, atlas guided, etc. are some of the examples of existing segmentations methods. In recent years, new variations of these segmentation methods have been proposed and utilized (Gerig et al., 2001; Li et al., 2008; Vicente et al., 2008; Chen et al., 2009; Ladicky et al., 2009; Vasilache et al., 2009; Chen et al. 2013). Nevertheless, each of these segmentation techniques has various drawbacks, for example, the shortcoming of thresholding technique is that it cannot be directly applied to multi-channel images (Sahoo et al., 1988). Region growing is sensitive to the choice of the initial seed (Haralick and Shapiro, 1985; Haralick and Shapiro, 1985). Some commonly used methods are clustering techniques such as K-means (Dunn, 1973; Coleman and Andrews, 1979; Yao et al., 2004), the fuzzy cmean algorithm (Lei and Sewchand, 1992), and the expectationmaximization algorithm (Liang et al., 1994; Rajapakse et al., 1997). However, clustering algorithms do not directly incorporate spatial modeling and can be sensitive to noise and intensity in homogeneities. In addition, the shape of the pelvic bones are quite complex. Typical CT scans obtained from each patient contain more than one hundred consecutive CT slices. Each CT slice includes several different bones with complicated and un-regular contours. In order to assist the radiologist diagnosing pelvic trauma, multiple pelvic bone structures need to be accurately segmented for each patient's data. Moreover, there are some other difficulties such as limited resolution of the original CT images and possible fractures of pelvic structures. CT images are also susceptible to noise, partial volume effects, and in-homogeneities. These make the segmentation process much more challenging and time-consuming. Furthermore, due to factors such as variations in bone tissues, complexity of pelvic structures, and significantly different geometrical characteristics of fractures, automatic segmentation of pelvic bone in CT scan remains as challenging tasks. Currently, there are no existing technologies with versatile capabilities to extract pelvic bone structure and provide satisfactory results. Hence developing an automated, efficient segmentation algorithm for the assessment of pelvic injuries can be a vital tool.

In this study, a new cross validation based segmentation algorithm is proposed to automatically extract multiple-level bone structures using a combination of anatomical knowledge and computational techniques. In order to test the accuracy of performance of the training models, a cross-validation process has been designed to indentify how segmentation can be affected by variations in training sets. In order to quantify the segmentation accuracy, two suitable measures are defined to evaluate the results: "mean distance" and "mis-segmented area." The results are promising, indicating that the proposed method can successfully detect the ilium, ischium, pubis and femur bones; also the quantitative measurements demonstrate the effectiveness and robustness of the proposed algorithm.

The rest of this study is organized as follows. Proposed method for bone segmentation is explained in section II, results are presented in section III followed by section IV, which presents conclusions and finally section $\mathrm{V}$ lists the future work.

\section{METHODS}

A. 2D Pelvic Bone Segmentation. Bone tissue segmentation by automated $\mathrm{CT}$ image processing can significantly reduce the time needed to examine medical images and to improve the accuracy of medical decision-making. However, automatic bone tissue segmentation from each CT image is very challenging due to the complexity of pelvic structures, and variation in bone structure from person to person. In addition, CT images are susceptible to noise, partial volume effects, and in-homogeneities. These make the bone segmentation more challenging and time-consuming.

This study describes a new cross validation based segmentation algorithm in multi-level pelvic CT scans, which can segment the pelvic bones from CT images automatically and accurately, as illustrated in Figure 1. The proposed method consists of five parts: preprocessing, edge detection, the best matching template detection, cross validation based registered active shape model (RASM) with automatic initialization and 3D pelvic model reconstruction.

Some of the prior work related to this study focuses on the segmentation and fracture detection of pelvic bones in CT scans (Smith and Najarian, 2009; Vasilache et al., 2009). As shown in Figure 1, the first step of segmentation is preprocessing. A multi-stage preprocessing technique is introduced, which includes surrounding artifacts filtering, morphological operations, and image enhancement. This step first removes the surrounding artifacts present in the original image, such as CT table, cables, hands, and lower extremities. High frequency speckle noise is also removed from the images using a median filter. Next, the image is enhanced to emphasize the features of interest - for example, the pelvic bone is enhanced to higher intensity while less emphasis is placed on soft tissues and other organs. Once image enhancement is conducted using brightness contrast stretching, a series of steps are applied for preliminary bone tissue segmentation, including Canny edge detection technique to detect the edges of bone tissue, morphological operations to remove spurious edges and sub-edge connections and removal. The obtained preliminary segmented results are then used for detecting the best matching template. To design the segmentation process in an automated fashion, it is performed by a template-based best shape matching algorithm (Belongie et al., 2002). For this technique, 100 bone templates are created through manual selection from the visible human project dataset (http://www.nlm.nih.gov/research/visible/visible_human.html), and these templates are compared to each CT slice in order to determine the best matched template, which allows the corresponding training shape models of each best matched template to be applied to the detected edge for bone segmentation. One important consideration is that the pelvic bones in each of the original training images have different sizes, rotation angles and locations that may lead to unstable and unreliable shape models for inaccurate bone segmentation. Hence to overcome this, a method called

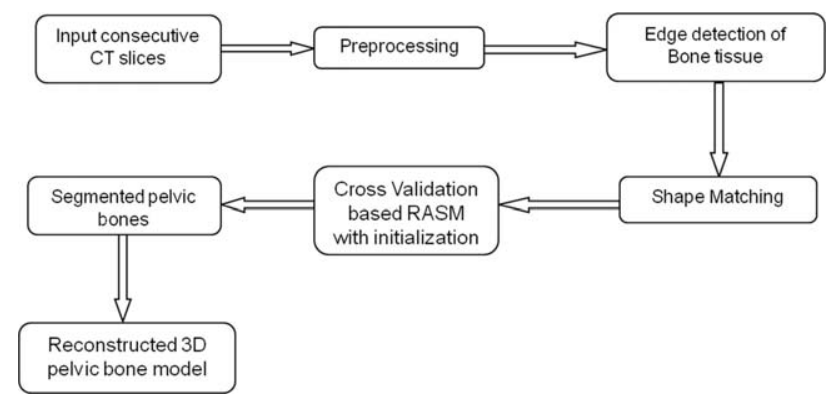

Figure 1. Schematic diagram of pelvic bone segmentation. 


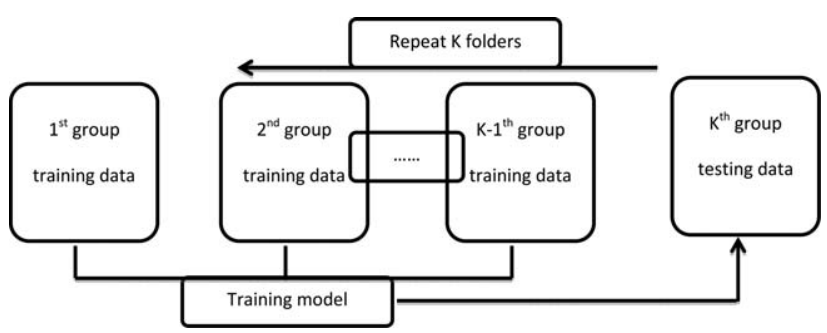

Figure 2. Flowchart of Traditional cross-validation.

registered active shape model (RASM) is used for the final bone segmentation (Wu et al., 2011). By using this method to extract pelvic bone tissues, a more robust training model is developed as compared to models developed using standard active shape model (ASM) algorithms.

ASM (Cootes et al., 1995) is a supervised learning technique that requires a set of labeled training images. The structure of interest in these images is represented by a series of landmarks manually placed along its boundaries. The statistical model of the desired structure is formed by extracting the shape and intensity information across the training set. This model is used to evaluate the possible deformation of each structure as the algorithm searches for the same structure in the test new images. The first step in constructing the shape model is to determine a set of $n$ landmarks, which describe the structure of interest in every training image. A given object can be represented as a $2 n$ element vector $x$ given by $x=\left[x_{1}, \ldots, x_{n}, y_{1}, \ldots y_{n}\right]$, where $\left(x_{i}, y_{i}\right)$ provides the coordinates of landmark $x$. ASM uses an iterative approach that sets the model parameters $b$ to the mean shape of $x$, that is, $\bar{x}$. Principle component analysis (PCA) is used to reduce the dimensionality of the dataset. $x_{i}$ can be approximated by:

$$
x_{i} \approx \bar{x}+P b
$$

where, $P$ contains the eigenvectors of the PCA covariance matrix. Shape in this frame is defined by $b ; x$ is aligned with a new set of image points $Y$ (representing the shape in a new image). The image points are then projected into the model space so that the parameters in $b$ can be updated to match them. The process is repeated until there is no significant change in $b$ between iterations.

The entire RASM algorithm includes two stages: training stage in which registered training models are created, and the testing stage that includes automatic initialization. During the training stage of segmentation, for each diverse shape of the pelvic bones in different slices, distinct numbers of landmarks are placed on those boundaries. Here shows different numbers of landmarks for each pelvic bone: lumbar (23-27), ilium (30-40), sacrum (8-10), femur (20-22), pubis (20-24), ischium. The numbers of the landmarks vary depending on the size of each bone structure in different training sample set. The structure of interest in these images is represented by a series of landmarks manually placed along its boundaries by the expert. The statistical model of the desired structure is formed by extracting the shape and intensity information across the training set. This model is used to evaluate the possible deformation of each structure as the algorithm searches for the same structure in the new test images. During the test stage, the landmarks will move towards the target position so that the parameters of the algorithm will be optimized and updated until there is no significant change between iterations.

ASM takes a statistical approach that requires a set of labeled training images to determine variations of the desired shape in testing the new images. The standard ASM has been widely used in the recent years, but this method is highly sensitive to initialization. It requires that the initial position be correctly assigned to the training model in order to detect a target object in the image. Then the algorithm can attempt to fit the shape model to the object. If the shape model is not accurately placed, the standard ASM method often fails to detect the target object.

This study addresses this shortcoming using a hierarchical initialization process that composes image registration, extracted bone features as well as prior edge detection results to sequentially place the training models for each individual object. This process avoids the need for manually indicating the initial positions. This will avoid human interference and reduce human-caused errors. The algorithm in detail is described in (Wu et al., 2011). In short, the method makes use of the sorted centroids of mean shapes in training models and testing images, produce the paired up training model and testing bone shapes, then assigns the initial position of the mean shapes to the uppermost position of the testing bone.

B. Considerations During Training. As ASM (Cootes et al., 1995) is a supervised learning method, we are required to test the reliability of the performance of training models. A cross-validation based method is designed to test how much different training data can affect the segmentation results.

In traditional $K$-fold cross-validation, the original sample dataset is randomly divided into $K$ groups. A single group is retained as the testing data for validating the performance of the training model, and the remaining $K-1$ groups of data are used to create the training model. The cross-validation process is then repeated until each of the $K$ subsamples are used once as the validation data. Finally, the $K$ results can be averaged or combined to produce a single estimation as the final result. Figure 2 shows the mechanism of how traditional cross-validation works.

In our designed cross-validation method for pelvic bone models, the entire dataset (20 patients) are randomly assigned to four distinct groups. These groups are not representative of anything in specific; rather it is merely a mechanism to perform the leave one group out cross validation process. Since there are 20 subjects' datasets, each of the four groups contains five datasets. Different from traditional cross-validation method, within the each group of data, for each round, one subject is selected from a particular group for testing. The remaining subjects in the other three groups are used for creating three different training models, which apply to each selected testing subject. This process is designed to avoid choosing overlapping data for creating different training models and thus making them independent of each other. Figure 3 shows the mechanism of how our designed cross-validation method works. Different key pelvic bone structures, including right ilium, left ilium, right femur, left femur, right pubis and left pubis are segmented to demonstrate and compare the performance of training models.

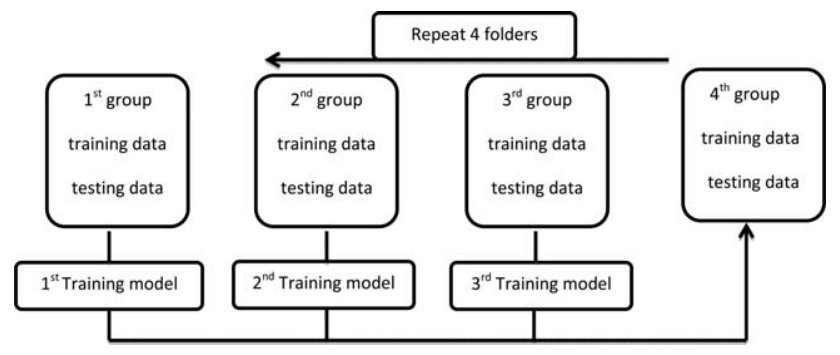

Figure 3. Flowchart of Designed cross-validation. 


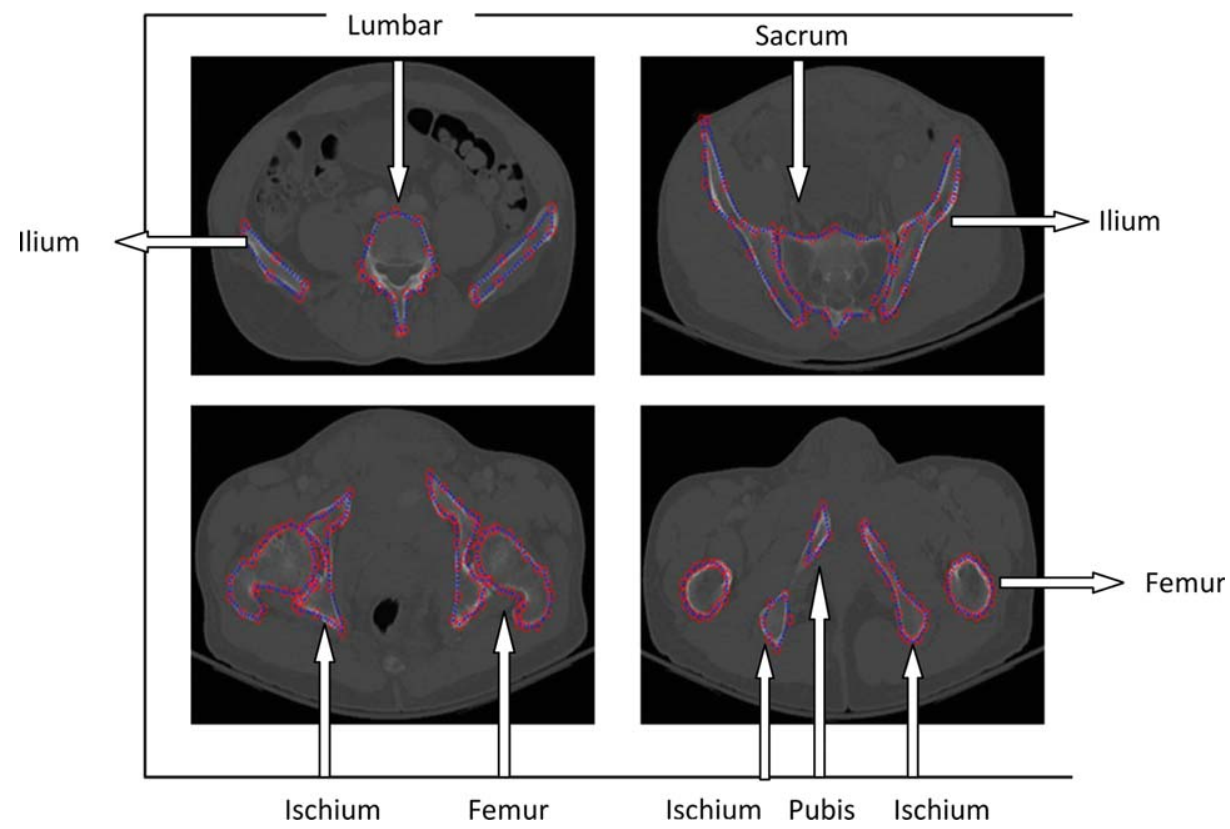

Figure 4. Pelvic bone segmentation results.

C. Evaluation Measures. These results were evaluated by expert radiologist as ground truth for assessment. Visual inspection is also used for evaluating the performance of pelvic bone segmentation. The segmented bones are classified into three categories: accurate, fair, and unacceptable. These categories were determined via consultation with a trauma physician, who identified actual bone contour as the ground truth.

The computed value of mean distance can be used to classify the results in one of three classes. The shapes represented with mean distances of less than $1.6 \pm 0.2 \mathrm{~mm}$ are classified as "accurate," the shapes represented with mean distances between $1.6 \pm 0.2 \mathrm{~mm}$ and $2.2 \pm 0.2 \mathrm{~mm}$ are classified as "fair," the shapes represented with mean distances more than $2.2 \pm 0.2 \mathrm{~mm}$ are classified as "unacceptable." The segmentation result can also be classified into one of the three classes using the computed mis-segmented area value. The shapes represented with mis-segmented areas of less than $10 \%$ are classified as "accurate," the shapes represented with missegmented areas between 10 and $20 \%$ are classified as "fair," the shapes represented with mis-segmented areas more than $20 \%$ are classified as "unacceptable."

In order to quantify the segmentation accuracy, suitable measure is required to evaluate the segmentation results. Hence specific for this study, precise definitions for evaluation criteria have been developed those are useful and intuitive for evaluation purposes.

Mean Distance. Given two surfaces $S_{1}$ and $S_{2}$, in this study, $S_{1}$ is the ground truth of the pelvic bone surface as per consultation with a trauma physician; $S_{2}$ is the detected pelvic bone surface from our algorithm.

The distance $d\left(p_{n}, S_{2}\right)$ between two point on surfaces $S_{1}$ and $S_{2}$ are defined as:

$$
d\left(p_{n}, S_{2}\right)=\min _{p_{m} \in S_{2}}\left\|p_{n}-p_{m}\right\|_{2}
$$

The mean distance between surface $S_{1}$ and the surface $S_{2}$ as:

$$
d(\text { mean })=\frac{\sum_{n=1}^{L} d\left(p_{n}, S_{2}\right)}{L}
$$

where $p_{\mathrm{n}}$ denotes each landmark/location on surface $S_{1}$ and $p_{\mathrm{m}}$ denotes each landmark on surface $S_{2}, L$ denotes the total number of landmarks on the surface. During the evaluation stage, the ideal positions of landmarks on the gold standard surface of pelvic bone are determined by the expert to measure the accuracy of the segmentation. The landmarks are placed using the expert knowledge as the ground truth. Then, the suitable measures are made between the landmarks in test images and expected positions of the ground truth.

Mis-Segmented Area. The idea is to have a measure that represents the uncommon area of the segmented surface and gold standard surface of pelvic bone. After the bone structures have been segmented, the original images are set to the binary version. The areas of the objects can be determined in the binary image. The area here is defined as a value corresponding to the total number of pixels located within bone regions. Given two surfaces $S_{1}$ and $S_{2}$, we define the areas as $A_{1}$ and $A_{2}, A_{i}$ is the area for each bone surface $S_{i}$.the Mis-segmented Area $M_{A}$ of two surfaces $S_{1}$ and $S_{2}$ can be defined as:

$$
\begin{gathered}
\frac{|G|}{\left|A_{1}\right| * 100} \\
\text { where } G=\left\{\text { pixels }: p \mid p \in A_{1} \cup A_{2}, p \notin A_{1} \cap A_{2}\right\}
\end{gathered}
$$

D. 3D Pelvic Bone Visualization. Since 2D pelvic CT images provide limited and local information in each slice, 3D pelvic bone models are very often required to present a full range of the entire bone structure in clinical diagnosis. The radiologists usually observe and analyze the patients' data with the combination of both $2 \mathrm{D}$ images and 3D models to make accurate diagnostic decisions in clinical diagnosis, which is highly important for assessing the injury severity. After the pelvic bone from each continuous $2 \mathrm{D}$ pelvic CT image is successfully segmented, the 3D model is reconstructed utilizing these extracted 2D bone structures. In our work, the pelvic model can be rotated to any angle, which will help the physician comprehensively observe the entire pelvic bone structure and detect any abnormality of the patient, such as bone fractures. Detecting the presence and extent of the fracture is an important step in assessing 


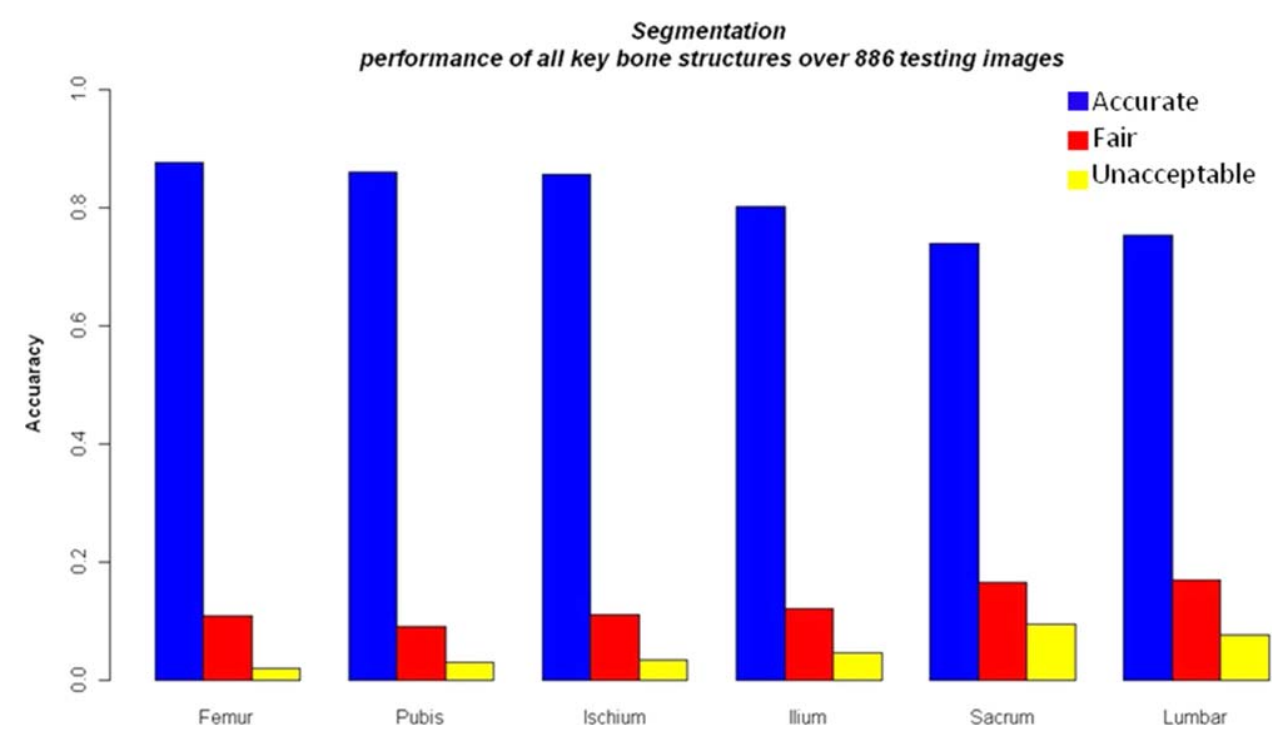

Figure 5. Segmentation accuracy results using proposed method of different pelvic bone structures. [Color figure can be viewed in the online issue, which is available at wileyonlinelibrary.com.]

the severity of a pelvic injury. There might be subtle fractures, as fractures of the acetabulum, hip displacement or presence of hemorrhage. In addition, 3D visualization may be used for further validating the 2D segmentation results. Here the isosurface method is applied to form the 3D pelvic bone models utilizing the segmented pelvic bones from 2D CT slices. In medical imaging area, isosurfaces is typically used to demonstrate regions of a specific density in a three-dimensional CT scan. This method allows the visualization of organs, bones, or other structures. An isosurface is a 3D surface that represents points of an equal constant value within a volume of space-3D data distribution. Isosurfaces are often used as data visualization methods and can be drawn on the screen very quickly.

After the $2 \mathrm{D}$ bone structures are extracted from continuous CT slices, a sphere in 3D domain is set as $\left\{x^{2}+y^{2}+z^{2}=R^{2}\right\}$. A threshold is set to 0.5 in order to create a binary version of the images. The $3 \mathrm{D}$ pelvic models can later be built based on the binary version of all CT slices. The pixel value of all the nonbone regions including the background, soft-tissue and all other organs represented in CT images are set to 0 , and the bone regions are set to 1 . An isofurface is therefore formed based on the threshold value to separate the bone region in each layer from the background. The isosurface connects the points that have the equal pixel value and represent a 3D model of pelvic bone structure.

E. Snake Method. In this section, we compare the segmentation performance using the proposed method and an automated seeding based Snake method (Kass et al., 1988). In Snake based methods, a deformable model is matched to a target in the image by means of energy minimization. A snake is initialized firstly near the target and the snake gets refined iteratively and moves

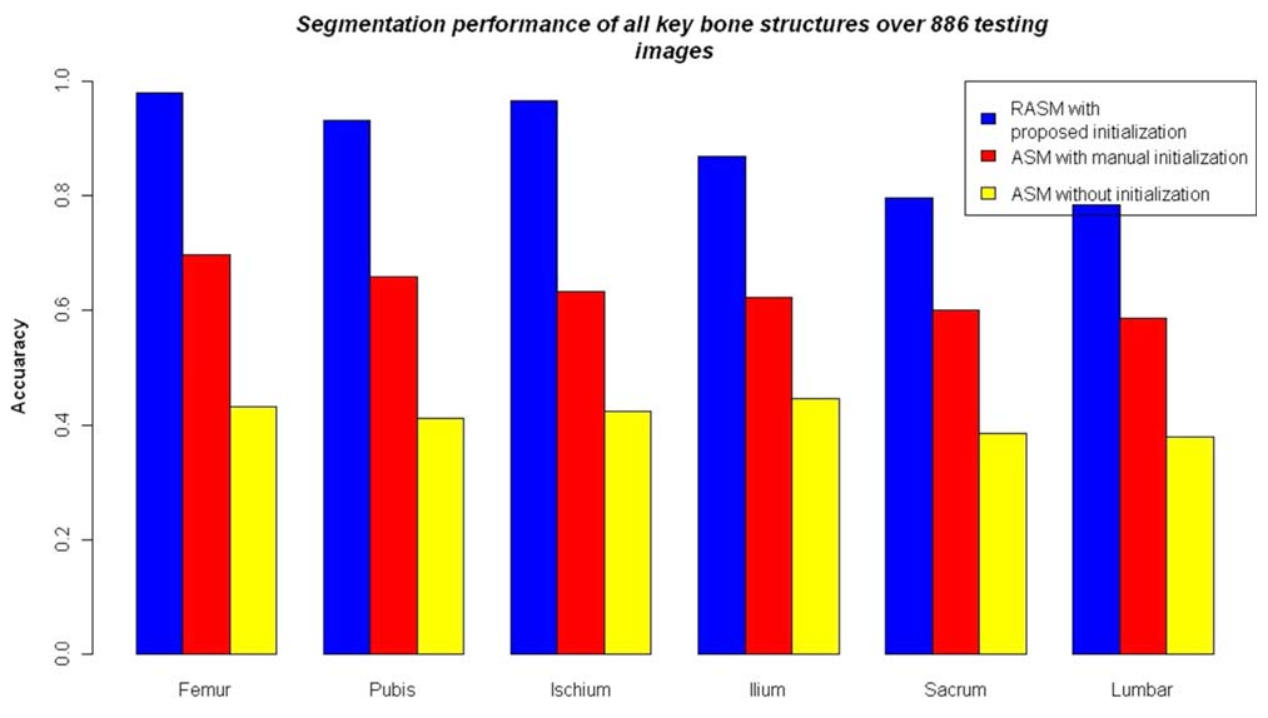

Figure 6. Segmentation accuracy including both accurate and fair results of different pelvic bones using proposed method, standard ASM with manual initialization and standard ASM without initialization. [Color figure can be viewed in the online issue, which is available at wileyonlinelibrary.com.] 


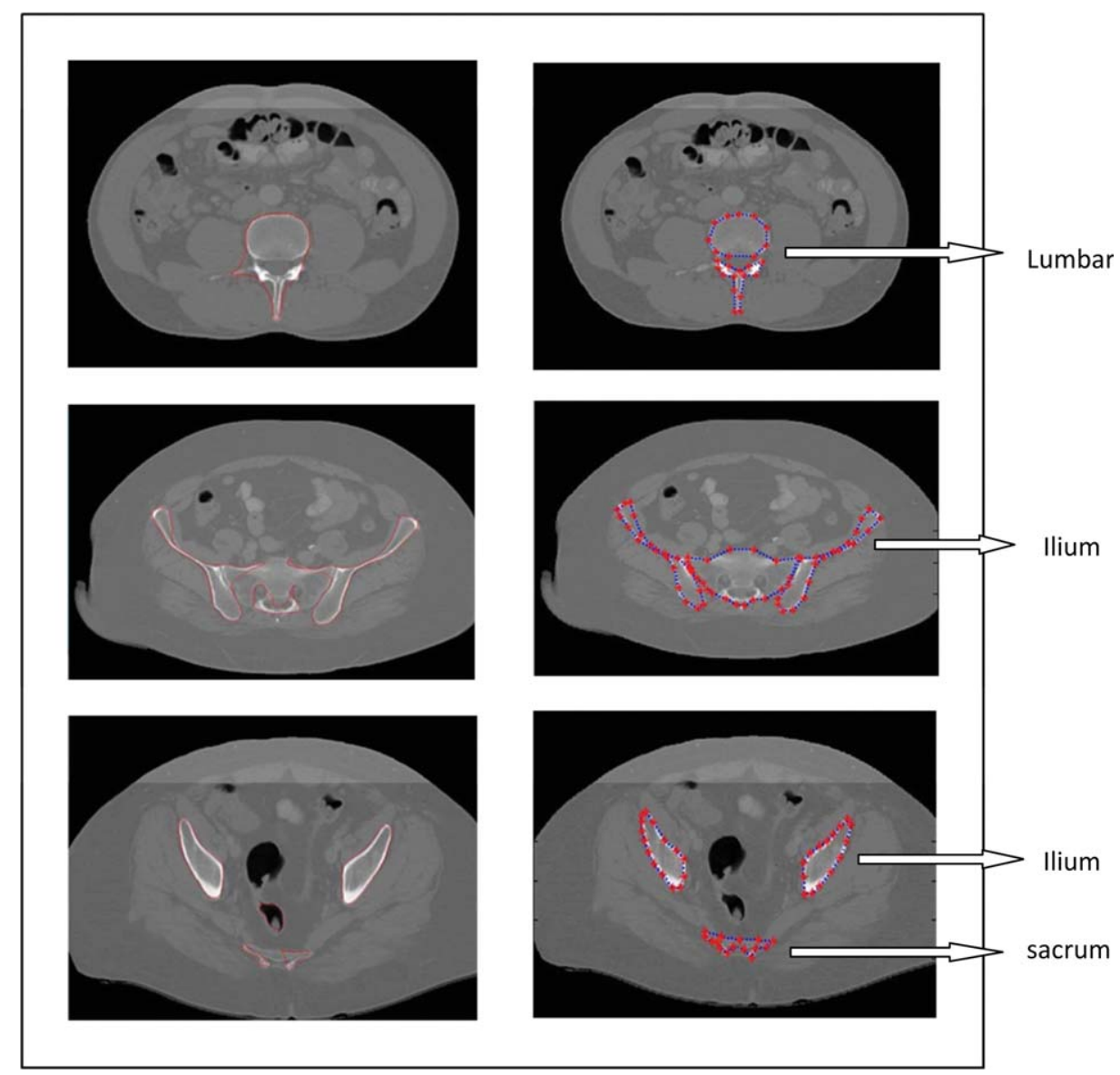

Figure 7. Compared results of pelvic bone segmentation via RASM and Snake methods.

towards the salient contour (Kass et al., 1988). The Snake method is often used in image segmentation. In snakes, a deformable model is matched to a target in the image by means of energy minimization. A snake is initialized firstly near the target and the snake gets refined iteratively and moves towards the salient contour.

The algorithm for the original Snake model was introduced by Kass et al. in 1988 (Kass et al., 1988). The optimal snake shape and position were defined based on the solution of minimizing the sum of the internal and external energies defined along the snake. The snake $S(s)$ is defined as a parameterized curve:

$$
S(s)=(x(s), y(s))
$$

The curve is evolved such that it minimizes the energy functional:

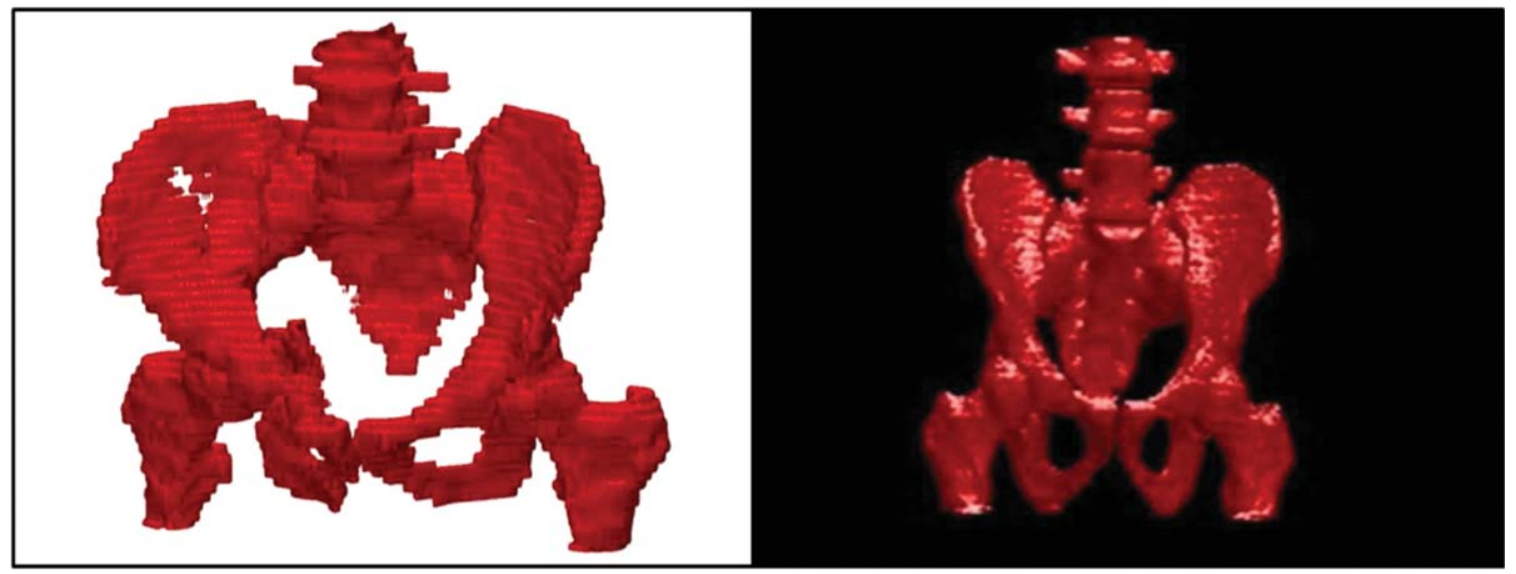

Figure 8. Example results of 3D pelvic bone structure. 
Table 1. Performance of three training models B-D for five testing subjects in A.

\begin{tabular}{llll}
\hline Testing Subjects/Training Model & Training Model B & Training Model C & Training Model D \\
\hline First testing subject in A & Accurate & Accurate & Accurate \\
& MD: $0.6 \pm 0.2 \mathrm{~mm}$ & MD: $1.0 \pm 0.2 \mathrm{~mm}$ & MD: $1.5 \pm 0.1 \mathrm{~mm}$ \\
Second testing subject in A & Accurate & Accurate & Accurate \\
& MD: $1.1 \pm 0.2 \mathrm{~mm}$ & MD: $1.2 \pm 0.3 \mathrm{~mm}$ & MD: $1.0 \pm 0.2 \mathrm{~mm}$ \\
Third testing subject in A & Accurate & Accurate & Accurate \\
& MD: $0.8 \pm 0.4 \mathrm{~mm}$ & MD: $1.0 \pm 0.5 \mathrm{~mm}$ & MD: $0.9 \pm 0.2 \mathrm{~mm}$ \\
Fourth testing subject in A & Fair & Accurate & Accurate \\
Fifth testing subject in A & MD: $1.7 \pm 0.6 \mathrm{~mm}$ & MD: $1.5 \pm 0.2 \mathrm{~mm}$ & MD: $1.3 \pm 0.3 \mathrm{~mm}$ \\
& Accurate & Accurate & Accurate \\
& MD: $0.8 \pm 0.2 \mathrm{~mm}$ & MD: $0.8 \pm 0.2 \mathrm{~mm}$ & MD: $0.9 \pm 0.4 \mathrm{~mm}$ \\
\hline
\end{tabular}

Table II. Performance of three training models A, C, and D for five testing subjects in B.

\begin{tabular}{llll}
\hline Testing Subjects/Training Model & Training Model A & Training Model C & Training Model D \\
\hline First testing subject in B & Accurate & Accurate & Accurate \\
& MD: $1.5 \pm 0.2 \mathrm{~mm}$ & MD: $1.6 \pm 0.3 \mathrm{~mm}$ & MD: $1.3 \pm 0.4 \mathrm{~mm}$ \\
Second testing subject in B & Accurate & Accurate & Fair \\
& MD: $0.6 \pm 0.2 \mathrm{~mm}$ & MD: $0.7 \pm 0.4 \mathrm{~mm}$ & MD: $2.2 \pm 0.8 \mathrm{~mm}$ \\
Third testing subject in B & Accurate & Accurate & Accurate \\
& MD: $1.6 \pm 0.3 \mathrm{~mm}$ & MD: $0.9 \pm 0.2 \mathrm{~mm}$ & MD: $1.2 \pm 0.2 \mathrm{~mm}$ \\
Fourth testing subject in B & Accurate & Fair & Accurate \\
& MD: $1.6 \pm 0.2 \mathrm{~mm}$ & MD: $2.1 \pm 0.7 \mathrm{~mm}$ & MD: $1.0 \pm 0.2 \mathrm{~mm}$ \\
Fifth testing subject in B & Accurate & Accurate & Accurate \\
& MD: $1.1 \pm 0.4 \mathrm{~mm}$ & MD: $0.8 \pm 0.2 \mathrm{~mm}$ & MD: $1.0 \pm 0.2 \mathrm{~mm}$ \\
\hline
\end{tabular}

Table III. Performance of three training models A, B, and D for five testing subjects in C.

\begin{tabular}{llll}
\hline Testing Subjects/Training Model & Training Model A & Training Model B & Training Model D \\
\hline First testing subject in C & Accurate & Accurate & Accurate \\
& MD: $1.2 \pm 0.4 \mathrm{~mm}$ & MD: $1.0 \pm 0.2 \mathrm{~mm}$ & MD: $1.3 \pm 0.3 \mathrm{~mm}$ \\
Second testing subject in C & Accurate & Accurate & Accurate \\
& MD: $0.8 \pm 0.2 \mathrm{~mm}$ & MD: $0.6 \pm 0.2 \mathrm{~mm}$ & MD: $0.6 \pm 0.2 \mathrm{~mm}$ \\
Third testing subject in C & Fair & Fair & Accurate \\
& MD: $2.2 \pm 0.5 \mathrm{~mm}$ & MD: $1.9 \pm 0.3 \mathrm{~mm}$ & MD: $1.6 \pm 0.3 \mathrm{~mm}$ \\
Fourth testing subject in C & Accurate & Accurate & Accurate \\
& MA: $1.6 \pm 0.3 \mathrm{~mm}$ & MA: $1.5 \pm 0.2 \mathrm{~mm}$ & MD: $1.3 \pm 0.4 \mathrm{~mm}$ \\
Fifth testing subject in C & Accurate & Accurate & Accurate \\
& MD: $0.7 \pm 0.2 \mathrm{~mm}$ & MD: $0.6 \pm 0.2 \mathrm{~mm}$ & MD: $1.0 \pm 0.1 \mathrm{~mm}$ \\
\hline
\end{tabular}

$$
E=\int_{0}^{1} \frac{\alpha}{2}\left|S^{\prime}(s)\right|^{2} d s+\int_{0}^{1} \frac{\beta}{2}\left|S^{\prime \prime}(s)\right|^{2} d s+\int_{0}^{1} F(s) d s
$$

$F(s)$ is the external energy which is derived from the given image in such a way that it takes smaller values around boundaries. $\alpha$ and $\beta$ are the parameters that control the curve's tension and rigidity, respectively.

Table IV. Performance of three training models A-C for five testing subjects in D.

\begin{tabular}{llll}
\hline Testing Subjects/Training Model & Training Model A & Training Model B & Training Model C \\
\hline First testing subject in D & Accurate & Accurate & Accurate \\
& MD: $1.1 \pm 0.4 \mathrm{~mm}$ & MD: $1.1 \pm 0.2 \mathrm{~mm}$ & MD: $1.2 \pm 0.3 \mathrm{~mm}$ \\
Second testing subject in D & Accurate & Accurate & Accurate \\
& MD: $1.0 \pm 0.2 \mathrm{~mm}$ & MD: $1.5 \pm 0.2 \mathrm{~mm}$ & MD: $1.4 \pm 0.4 \mathrm{~mm}$ \\
Third testing subject in D & Accurate & Accurate & Accurate \\
& MD: $0.8 \pm 0.2 \mathrm{~mm}$ & MD: $1.3 \pm 0.3 \mathrm{~mm}$ & MD: $0.9 \pm 0.4 \mathrm{~mm}$ \\
Fourth testing subject in D & Accurate & Accurate & Fair \\
& MD: $1.5 \pm 0.4 \mathrm{~mm}$ & MD: $1.6 \pm 0.2 \mathrm{~mm}$ & MD: $2.1 \pm 0.6 \mathrm{~mm}$ \\
Fifth testing subject in D & Accurate & Accurate & Accurate \\
& MD: $0.6 \pm 0.2 \mathrm{~mm}$ & MD: $0.7 \pm 0.3 \mathrm{~mm}$ & MD: $1.0 \pm 0.3 \mathrm{~mm}$ \\
\hline
\end{tabular}



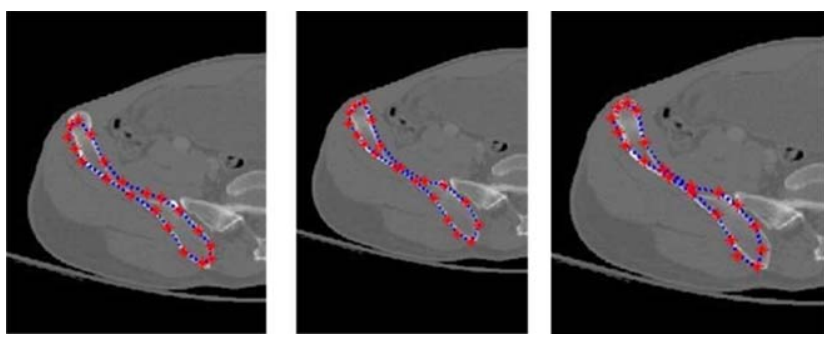

Figure 9. Results of pelvic ilium bone segmentation using three different training models for a testing subject. [Color figure can be viewed in the online issue, which is available at wileyonlinelibrary.com.]

Selecting appropriate seeds is very important for obtaining satisfactory segmentation result. This study presents a method of automatic initial seeds creation that is suitable for pelvic CT segmentation. Firstly initial bone mask is established using preprocessing, wavelet analysis and a suitable threshold. Next, refined bone mask is obtained using binary multiplication of the initial bone mask and the filtered image after applying Gaussian filtering. In order to form seeds that are close to the contour, Canny edge detection is utilized based on the refined mask and seeds are most likely located on the edge of the identified regions of interest.

Seed growing step consists of growing each seed from the refined mask in its own neighborhood. The established growing criterion is based on neighborhood grey level statistics and gradient values. A curve is initialized based on the initial seeds. Such initial curve evolves and moves through the image according to a solution that attempts to minimize the energy function (Vasilache and Najarian, 2009).

\section{RESULTS}

A. Dataset. The dataset has been obtained from the Virginia Commonwealth University Medical Center. Data has been collected from 20 patients with traumatic pelvic injuries. Anywhere between 45 and 129 images are collected for each patient, representing a variety of anatomical structures within the pelvic CT scans, especially those that display similar structures within the group of patients. Axial CT images with 1-5 mm slice thickness are used for the study. Scans collected from five patients are used for training while scans from the other fifteen patients are used for testing. In order to build appropriate training models for each bone structures, the expert's knowledge and experience is utilized to manually choose every landmark in each training image. In this study, it was found five different training images have been sufficient to express the variation of the couture for each bone structure. Also by adding more than five train-
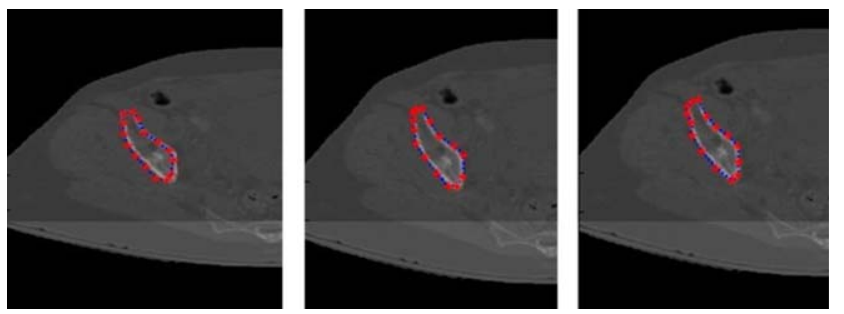

Figure 10. Results of pelvic ilium bone segmentation using three different training models for a testing subject. [Color figure can be viewed in the online issue, which is available at wileyonlinelibrary.com.]
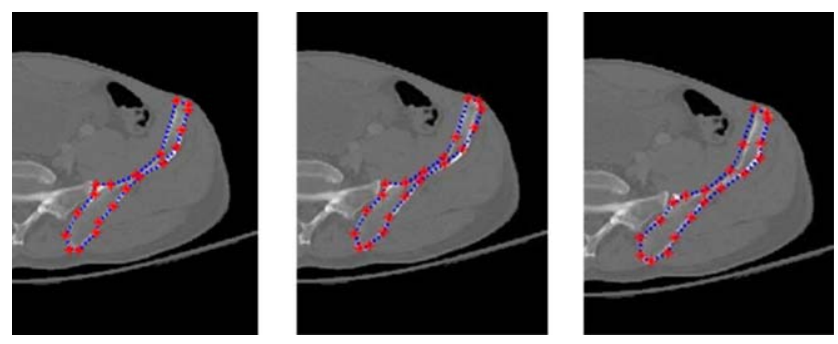

Figure 11. Results of pelvic ilium bone segmentation using three different training models for a testing subject. [Color figure can be viewed in the online issue, which is available at wileyonlinelibrary.com.]

ing images it was found that the performance of the algorithm became more time-consuming while not adding much to the segmentation accuracy. However, in our work, the number of the landmarks placed into the five training images is more than 30,000. Since all these landmarks are placed manually by the expert, if more training images were to be added then it would take the experts/physicians more time to insert thousands more landmarks into the training set, while not adding much to the overall segmentation performance. Hence, in order to achieve a suitable balance between the running speed and accuracy, five training images have been used in this work.

B. Results for Image Segmentation. Figure 4 shows the segmentation results based on RASM with the automatic initialization processing. The results show that the proposed method accurately segments the pelvic bones (lumbar, ilium, sacrum, femur, pubis, ischium).

Among all the segmentation results of 886 testing images across fifteen patients, $83.07 \%$ of them are classified as "accurate," and $13.54 \%$ of them are "fair" and $3.39 \%$ of them are detected to be "unacceptable." The total segmentation accuracy for both accurate and fair classes is $96.61 \%$

For different pelvic bone structures, the segmentation accuracy results are shown in Figure 5. The ilium, ischium, pubis and femur are almost always detected to be at least "fair"; however, the sacrum and lumbar show a number of unacceptable results. This may be because of the variation in bone shapes, blurred edge of the bones, poor quality of the original image, etc. The unacceptable results may be improved by further training of models across a wider dataset or using more landmarks for training the model. Segmentation accuracy, including both "accurate" and "fair" results of different pelvic bones using the proposed method, the Standard ASM with manual initialization and the Standard ASM without initialization, are shown
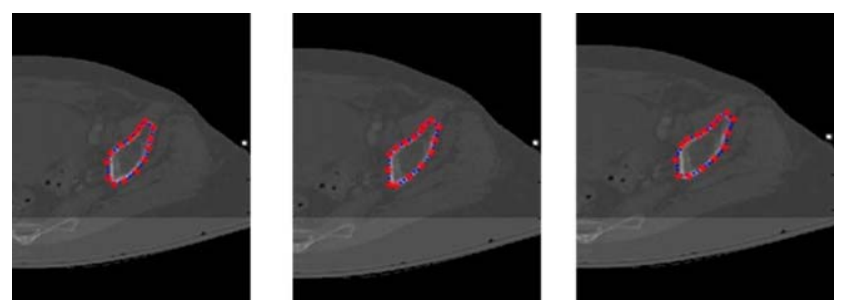

Figure 12. Results of pelvic ilium bone segmentation using three different training models for the second testing subject. [Color figure can be viewed in the online issue, which is available at wileyonlinelibrary.com.] 

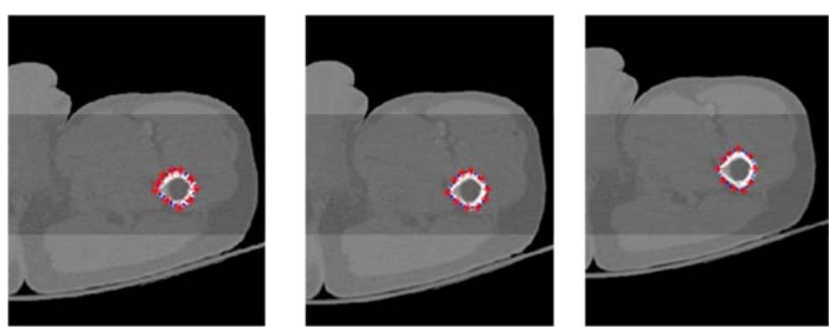

Figure 13. Results of pelvic femur bone segmentation using three different training models for a testing subject. [Color figure can be viewed in the online issue, which is available at wileyonlinelibrary.com.]

in Figure 6. The results show the superiority of the proposed method on all types of pelvic bones.

C. Results Compare with Snake Method. Figure 7 shows the compared results using the proposed method-RASM and the Snake method. The segmentation results using RASM are shown in the left side, the results using snake based method are shown in the right side. From the results, it can be seen that the Snake method detect two separated bones as one object. There are often pelvic bone structures which may be close to each other, for example, femoral head and ilium, where Snake method may fail to detect the separation between the bones. Also as can be seen in the third group results, the nonbone region is wrongly detected as bone structure using Snake.

D. Results for 3D Pelvic Bone Visualization. As shown in Figure 8 , the $3 \mathrm{D}$ structure of the pelvic bone is clearly presented from different points of view. The left image on Figure 6 clearly shows a cavity in the bone structure, where the detected fracture exists in the pelvic region. In this work the developed 3D pelvic model can be rotated to any angle which helps the physician comprehensively observe the entire pelvic bone structure and detect any abnormality of the patient. In addition, 3D visualization may be used for further validating the $2 \mathrm{D}$ segmentation results. However, for the specific measure of fracture or hemorrhage severity, 2D images should be utilized to provide more details.

\section{E. Results from One Group Based Cross}

Validation. Table 1 shows the average segmentation performance for the testing subjects in A using three training models created from $\mathrm{B}$ to D. Table II presents the average segmentation performance for the testing subjects in B using three training models created from A, $\mathrm{C}$, and D. Table III shows the average segmentation performance for
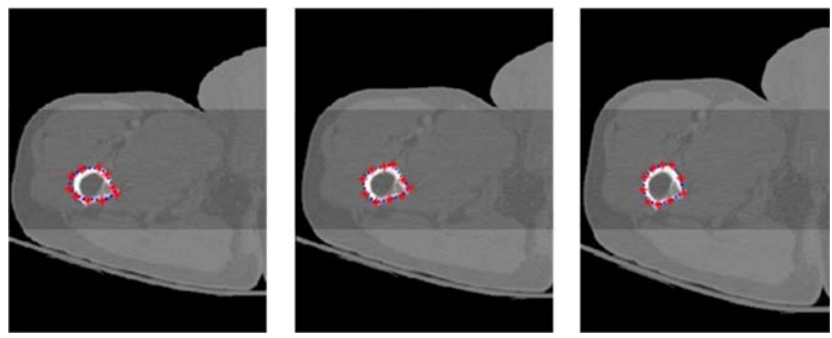

Figure 14. Results of pelvic femur bone segmentation using three different training models for a testing subject. [Color figure can be viewed in the online issue, which is available at wileyonlinelibrary.com.]
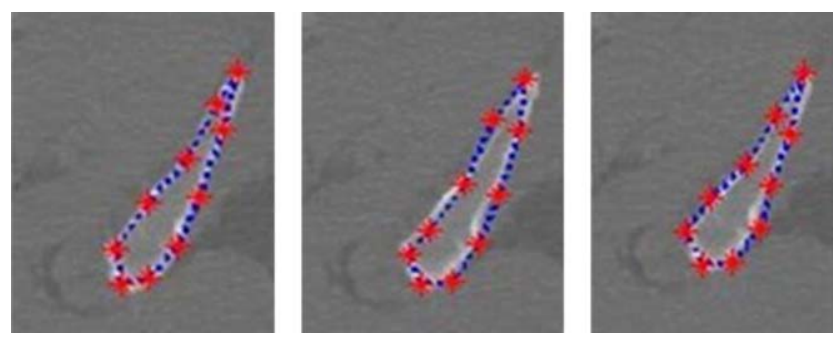

Figure 15. Results of pelvic pubic bone segmentation using three different training models for a testing subject. [Color figure can be viewed in the online issue, which is available at wileyonlinelibrary.com.]

the testing subjects in $\mathrm{C}$ using three training models created from $\mathrm{A}$, $\mathrm{B}$, and D. Table IV presents the average segmentation performance for the testing subjects in D using three training models created from A to C. In the tables, MD denotes Mean Distance between the segmented surface and ground truth surface.

Figures 9 and 10 show the example results of left pelvic ilium bone segmentation using three different training models for two testing subjects. Figures 11 and 12 present the example results of left pelvic ilium bone segmentation using three different training models for two testing subjects. Figure 13 shows the example results of right pelvic femur bone segmentation using three different training models for a testing subject. Figure 14 shows the example results of left pelvic femur bone segmentation using three different training models for a testing subject. Figure 15 shows the example results of right pelvic pubic bone segmentation using three different training models for a testing subject. Figure 16 shows the results of left pelvic pubic bone segmentation using three different training models for a testing subject.

For the segmentation performance, $90 \%$ of the total testing subjects are classified as accurate and there is slight difference among the segmentation results using different training models. Based on the entire performance of three different training models on key pelvic bone structures across testing subjects, we can conclude that the selection of training data to form training models has slight influence to the final segmentation results. Also, each training model performs well on different testing subjects and most of the segmentation results are classified as the accurate. The created training models in this study for pelvic bone segmentation are effective and reliable.

\section{CONCLUSIONS}

This study provides a framework for the pelvic bone segmentation and $3 \mathrm{D}$ pelvic bone visualization. Pelvic CT scans of 20 patients are processed and bone structures from fifteen patients are accurately and automatically segmented using the proposed hierarchical
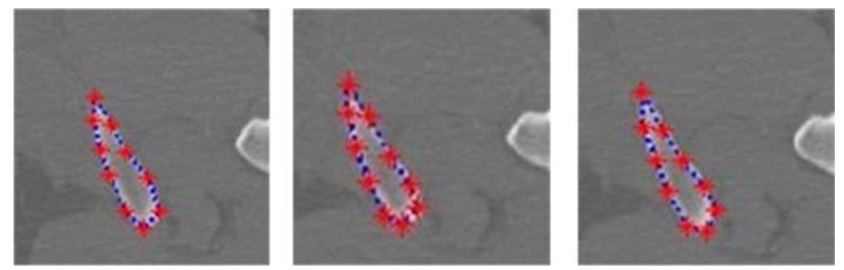

Figure 16. Results of pelvic pubic bone segmentation using three different training models for a testing subject. [Color figure can be viewed in the online issue, which is available at wileyonlinelibrary.com.] 
approach which incorporates preprocessing, edge detection, shape matching, homogeneity based image registration and RASM with novel initialization. Segmentation results are evaluated using two quantitative measures and the compared results show that the proposed method performs better with higher segmentation accuracy than the standard ASM. Also, compared results indicate that the proposed method performs better than Snake algorithm. Additionally, computation time taken by this method is less than the manual segmentation, making it practically applicable in automated processing of medical images. Different training models are created to segment key pelvic bone structures and the results show that the selection of training data does not have a large affect on the final segmentation performance. 3D pelvic bone models are built based on 2D segmentation results.

\section{FUTURE WORK}

Future work includes the following items: Process a larger database, with higher resolution CT images to improve the performance of the algorithm in segmenting the bones and detecting the bone fracture. Explore 3D bone segmentation algorithms based on pelvic CT images with higher resolution. Apply an appropriate rule-based methodology to generate rules for outcome prediction. This will form a decision-making system to provide physicians with reliable recommendations on diagnosis as well as treatment planning.

\section{ACKNOWLEDGMENTS}

The dataset used for this project is provided by Virginia Commonwealth University Medical Center.

\section{REFERENCES}

S. Ali, T. Pedro, D. Joseph, O. Marcus, I. Kenji, M.R. Daniel, and D. Demetrios, Predictors of positive angiography in pelvic fractures: A prospective study, J Am Coll Surgeons 207 (2008), 656-662.

S. Belongie, J. Malik, and J. Puzicha, Shape matching and object recognition using shape contexts, IEEE Trans Pattern Anal Mach Intell 24 (2002), 509-522.

Bureau of Labor Statistics, National census of fatal occupational injuries in 2011, U.S. Department of Labor, September 2012.

CDC Report, Access to trauma centers in the United States, September 2009.

W. Chen, R. Smith, S.Y. Ji, K. Ward, and K. Najarian, Automated ventricular systems segmentation in brain CT images by combining low-level segmentation and high level template matching, BMC Med Inform Decis Making 9 (Suppl 1) (2009), S4.

W. Chen, C. Cockrell, K. Ward, and K. Najarian, Predictability of intracranial pressure level in traumatic brain injury: Features extraction, statistical analysis and machine learning based evaluation, J Data Mining Bioinform 8 (2013), 480-494.

G.B. Coleman and H.C. Andrews, Image segmentation by clustering, Proc IEEE 5 (1979), 773-785.

T.F. Cootes, C.J. Taylor, D.H. Cooper, and J. Graham, Active shape models-Their training and application, Comput Vis Image Understand (1995), 38-59.

J.C. Dunn, A fuzzy relative of the ISODATA process and its use in detecting compact well-separated clusters, J Cybernet 3 (1973), 32-57.

G. Gerig, M. Jomier, and M. Chakos, Valmet: A new validation tool for assessing and improving 3D object segmentation, Medical Image Comput- ing and Computer-Assisted Intervention-MICCAI 2001 Lecture Notes in Computer Science 2208 (2001), 516-523.

R.M. Haralick and L.G. Shapiro, Image segmentation techniques, Comput Vis Graph Im Proc 29 (1985), 100-132.

S.Y. Ji, R. Smith, T. Huynh, and K. Najarian, A comparative analysis of multi-level computer-assisted decision making systems for traumatic injuries, BMC Med Inform Decis Making (2009) 9: p2.

M. Kass, A. Witkin, and D. Terzopoulos, Snakes: Active contour models, Int J Comput Vis (1988) 1: 321-331.

L. Ladicky', C. Russell, P.Kohli, and P.H.S. Torr, Associative hierarchical CRFs for object class image segmentation, IEEE International Conference on computer vision, Kyoto, Japan, 2009, pp. 739-746.

T. Lei and W.Sewchand, Statistical approach to X-ray CT imaging and its applications inimage analysis . II. A new stochastic model-based image segmentation technique for X-ray CTimage, IEEE Trans Med Imaging 11 (1992), 62-69.

C. Li, C. Kao, J.C. Gore, and Z. Ding, Minimization of region-scalable fitting energy for image segmentation, IEEE Trans Image Process 17 (2008), 1940-1949.

Z. Liang, J.R. MacFall, and D.P. Harrington, Parameter estimation and tissue segmentation frommultispectral MR images, IEEE Trans Med Imaging 13 (1994), 441-449.

Z. Peter, V. Bousson, C. Bergot, and F. Peyrin, A constrained region growing approach based on watershed for the segmentation of low contrast structures in bone micro-CT images, Pattern Recogn 41 (2008), 2358-2368.

J.C. Rajapakse, J.N. Giedd, and J.L. Rapoport, Statistical approach to segmentation of single-channel cerebral MR images, IEEE Trans Med Imag 16 (1997), 176-186.

P.K. Sahoo, S. Soltani, and A.K.C. Wong, A survey of thresholding techniques, Comput Vis Graph Im Proc 41 (1988), 233-260.

M.A. Schiff, A.F. Tencer, and C.D. Mack, Risk factors for pelvic fractures in lateral impact motor vehicle crashes, Accident Anal Prevent 40 (2008), 387-391.

R. Smith and K. Najarian, A hierarchical method based on active shape models and directed hough transform for segmentation of noisy biomedical images; application in segmentation of pelvic X-ray images, BMC Med Inform Decis Making, 9 (Suppl 1) (2009), S2.

UMD, Ciren report: Consequences and costs of lower-extremity injuries, EMS University of Maryland National Study Center for Trauma, 2005.

S. Vasilache and K. Najarian, A unified method based on wavelet filtering and active contour models for segmentation of pelvic CT images, ICME International Conference on complex medical engineering, 2009.

S. Vasilache, K. Ward, and K. Najarian, Unified wavelet and Gaussian filtering for segmentation of CT images; application in segmentation of bone in pelvic CT images, BMC Med Inform Decis Making, 9 (Suppl 1) (2009), S8.

S. Vicente, V. Kolmogorov, and C. Rother, Graph cut based image segmentation with connectivity priors, IEEE conference on computer vision and pattern recognition, Anchorage, Alaska, USA, 2008, pp 1-8.

J. Wu, P. Davuluri, K. Ward, C. Cockrell, R. Hobson, and K. Najarian, A new hierarchical method for multi-level segmentation of bone in pelvic CT scans, IEEE Engineering in Medicine and Biology Society (EMBC '11, oral), Boston, USA, 2011, pp. 3399-3402.

J. Yao, M. Miller, M. Franaszek, and R.M. Summers, Colonic plyp segmentations in CT colonography-based on fuzzy clustering and deformable models, IEEE Trans Med Imaging 23 (2004), 1344-1352.

J.W. Young, A.R. Burgess, R.J. Brumback, and A. Poka, Pelvic fractures: Value of plain radiography in early assessment and management. Radiology 160 (1986), 445-451. 